\title{
Sonography of Fetal Midgut Herniation: Normal Size Criteria and Correlation with Crown-Rump Length
}

\author{
Richard A. Bowerman, MD
}

Forty-eight fetuses were prospectively evaluated to (1) determune the normal size range of herniated midgut and (2) correlate sonographically the timing of midgut herniation with CRL.

All fetuses of CRL $38 \mathrm{~mm}$ or less, and two of six fetuses with a CRL of 40 to $42 \mathrm{~mm}$, demonstrated midgut herniation. No fetuses with a CRL greater than $44 \mathrm{~mm}$ had this finding. The cord containing midgut increased roughly from 4 to $7 \mathrm{~mm}$, in maxi- mum dimensions, for CRL of 19 to $41 \mathrm{~mm}$. An anterior abdominal wall mass greater than $7 \mathrm{~mm}$ at any CRL, or of any size in a fetus of CRL greater than 44 $\mathrm{mm}$, is suggestive of a fetal anomaly. Alternatively, a cord base "mass" within the 4 to $7 \mathrm{~mm}$ range for a CRL of 19 to $44 \mathrm{~mm}$ can be considered normal and not to require any follow-up. KEY WORDS: Fetus, gastrointestinal tract; Fetus, growth and development; Fetus, ultrasound studies.
$\mathrm{D}$ etailed sonographic visualization of the first trimester fetus often depicts a normal stage of intestinal tract development in which the midgut herniates into the base of the umbilical cord, creating an echogenic "mass." This must be recognized as a normal embryologic occurrence and differentiated from a pathologic fetal anterior abdominal wall mass. Although the sonographic recognition of midgut herniation has been reported, concern exists that an early diagnosis of an anterior wall defect may be impossible because of the potential overlap in its sonographic appearance with physiologic midgut herniation. ${ }^{1-4}$

The goals of this study were twofold: (1) to corre-

\section{ABBREVIATIONS}

CRL, Crown-rump length

Received June 12, 1992, from the Department of Radiology, University of Michigan Medical Center, Ann Arbor, Michigan. Revised manuscript accepted for publication November 19, 1992.

Address correspondence and reprint requests to Richard A. Bowerman, MD, University of Michigan Medical Center, Department of Radiology, Box 0030, Ann Arbor, MI 48109-0030. late sonographically the timing of midgut return to the abdomen with CRL or gestation age and (2) to determine the normal size range of the cord containing herniated midgut throughout its sojourn in the umbilical cord.

The establishment of sonographic criteria derived from a knowledge of both the sonographically detectable timing of midgut herniation relative to the CRL and its expected size at any given CRL or gestational age should allow distinction between most abdominal wall defects and this physiologic process.

\section{MATERIALS AND METHODS}

Forty-eight fetuses with CRL ranging from 19 to 82 $\mathrm{mm}$ ( 8.4 to 14.0 weeks' gestational age), in whom the anterior abdominal wall could be well imaged, were included prospectively in the study. Imaging was accomplished by the author in most instances, using commercially available high resolution real-time equipment, employing $3.5 \mathrm{MHz}$ or occasionally 5.0 $\mathrm{MHz}$ probes. Except in one instance, all scanning was performed transabdominally.

Scans in both transverse and sagittal planes were 
obtained through the fetal abdomen at the level of the umbilical cord insertion, with care taken to maximize visualization of the longest and widest segment of cord arising from the fetus and to confirm that the actual cord base at its point of attachment to the fetal abdomen was being imaged. Herniated midgut was identified as an echogenic area or "mass" in the base of the umbilical cord, adjacent to the fetal abdominal wall. The maximum transverse and longitudinal dimensions of the umbilical cord, where it contained echogenic midgut, were documented and measured. The single assigned size of the midgut herniation for a given fetus was the largest value determined by this method. Absence of herniated bowel was confirmed when the parallel linear echoes of vessel walls in an umbilical cord of uniform caliber could be traced to the anterior abdominal wall without any other echoes being present.

The CRL was documented and measured in all cases, and a gestational age was derived from the CRL using the data of Robinson and Fleming. ${ }^{5}$

\section{RESULTS}

Sonographically, physiologic midgut herniation was recognized as a solid mass of mid to high level echogenicity within the base of the umbilical cord adjacent to the fetal anterior abdominal wall. This appearance was in contrast to the umbilical cord itself, which was identified primarily by the visualization of bright, parallel linear echoes representing specular reflections from the external surface of the cord and the walls of its contained vessels. The shape of the cord containing the herniated midgut was spherical to slightly oblong with its lengthwise dimension generally equal to or greater than its width at the abdominal wall insertion.

The size of the umbilical cord, while it contained the herniated midgut, ranged in a linear fashion from a maximum dimension of $4.0 \mathrm{~mm}$ at a CRL of $19 \mathrm{~mm}$ to $7.0 \mathrm{~mm}$ at a CRL of $41 \mathrm{~mm}$ (corresponding to 8.4 to 10.8 weeks' gestation age) (Fig. 1).

Figure 1 shows the status of midgut herniation relative to CRL. It was documented in all instances in which the CRL was less than or equal to $38 \mathrm{~mm}$. In all cases in which the CRL was greater than or equal to $45 \mathrm{~mm}$, the midgut had returned to the abdominal cavity. For crown-rump lengths of 40 to $42 \mathrm{~mm}$, the midgut was in a variable position, with continued herniation in two instances and reduction in another four cases.

Summarized in terms of gestation age, Figure 2 shows the number of fetuses at specific gestation ages in whom the midgut was within the cord, and those

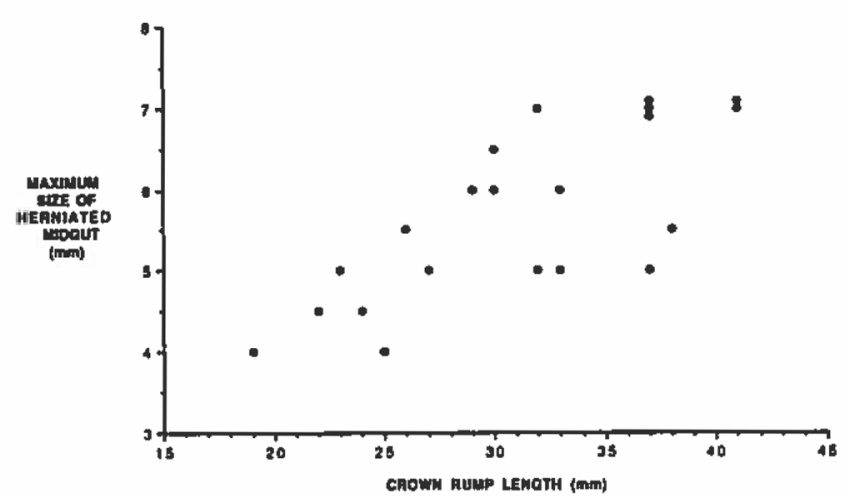

Figure 1 Size of herniated midgut relative to CRL.

showing midgut return to the abdominal cavity. In all 20 fetuses between 8.4 and 10.5 weeks' gestation, the midgut was seen within the cord. In the transition period from 10.6 to 10.9 weeks, only two of six fetuses had midgut documented in the cord. The 20 fetuses of greater than 11.1 weeks' gestation age all showed return of the midgut to the fetal abdomen.

\section{DISCUSSION}

According to Moore, 6 during the sixth week of embryologic development, the elongating midgut herniates into the proximal umbilical cord, owing to a relative shortage of intra-abdominal space. Within the cord, the midgut grows further and commences rotation about the superior mesenteric artery. During the tenth embryologic week (twelfth gestational week), the midgut returns to the now more capacious abdominal cavity, to complete rotation and fixation to the posterior abdominal wall.

This prospective study includes fetuses throughout the first trimester, both with and without midgut herniation, to address more precisely the timing of midgut herniation relative to CRL and gestation age, as defined with ultrasonography. The sonographi-

Figure 2 Location of midgut for given gestational age.

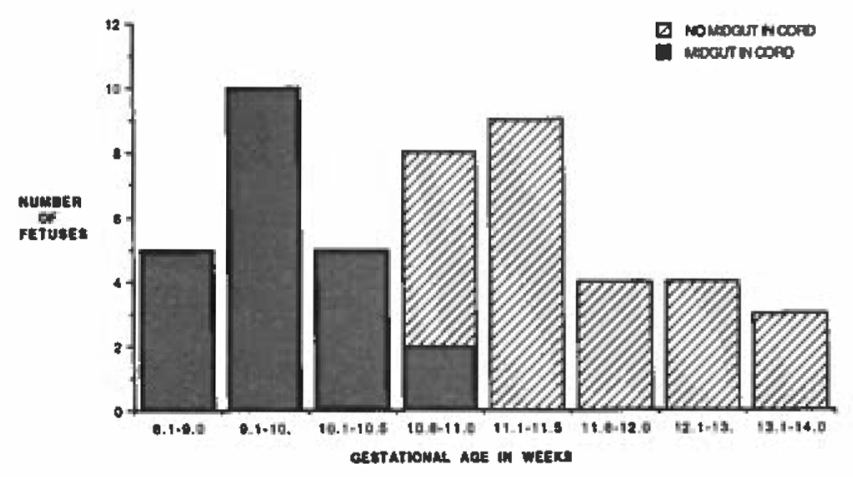


cally observed presence or absence of midgut within the cord correlates closely with the embryologic descriptions of the process. The sonographic findings of no herniated midgut in fetuses of over $44 \mathrm{~mm}$ CRL (11.2 weeks' gestational age) also correlate very closely with the pathologic evaluation of 41 first trimester abortuses documented by $\mathrm{Cyr}$ and associates, ${ }^{1}$ in which all midgut herniations were reduced by 11 weeks' gestational age.

Schmidt and coworkers 7 studied 14 patients serially between 7 and 12 weeks, also confirming return of the midgut to the abdomen between 10 and 12 weeks. The aim of their study was to evaluate how early midgut herniation could be detected, and the timing for its reduction was not narrowed down. The overall range is consistent with the results of this study and embryologic findings, however. In contrast, Green and Hobbins ${ }^{8}$ reported a $20 \%$ rate of persistent herniation at 12 weeks. The explanation for this approximately 1 week discrepancy is not apparent, but it clearly is at variance with embryologic descriptions and other sonographic studies.

Employing transvaginal scanning, Timor-Tritsch and colleagues ${ }^{9}$ observed midgut herniation in all fetuses at 9 to 10 weeks, in two of eight fetuses at 11 weeks, and in no 12 week fetus. These findings are consistent with those in the current study and the fetal and embryologic results cited here. However, the limited flexibility inherent in transvaginal scanning might make identification and measurements of maximum herniated midgut dimensions difficult. ${ }^{10}$

Cyr and associates ${ }^{1}$ retrospectively described the echogenic appearance of fetal midgut herniation seen in 10 cases between 7 and 10 gestation weeks, noting a size range of 5 to $10 \mathrm{~mm}$ (mean, $6.3 \mathrm{~mm}$ ). These dimensions are somewhat greater than the 4 to $7 \mathrm{~mm}$ range defined in the current study, perhaps relating to the retrospective nature of evaluation, as exact cord margins may not have been clearly apparent.

The size of the herniated midgut also was noted in 14 patients by Schmidt and coworkers. ${ }^{7}$ For fetuses at 9 weeks it measured 5 to $6 \mathrm{~mm}$, similar to the results of the current study. They found the herniated mass to measure 6 to $9 \mathrm{~mm}$ at 8 weeks, however, a finding at considerable odds with the current study as well is embryologic descriptions of an increasing size of the growing midgut during its herniated phase. Considering the small embryo size (CRL $=16 \mathrm{~mm}$ ) at 8 weeks, a diameter measurement of $9 \mathrm{~mm}$ for the herniated midgut is unlikely and probably includes nonmidgut structures. The major differential diagnosis of fetal anterior abdominal wall masses includes omphalocele, gastroschisis, and rarely such entities as cloacal or bladder exstrophy, pentalogy of Cantrell, and limb-body wall complex. The rare anoma- lies manifest extensive wall defects, which are readily defined as pathologic. The paraumbilical locus of involvement with gastroschisis, preserving a normal cord insertion, should exclude this entity from consideration. "The primary differential consideration for physiologic herniation is therefore an omphalocele. However, utilizing the size and timing criteria defined in this study, only the smallest of omphaloceles would not be separable from normal midgut herniation. The majority of omphaloceles contain liver, which has an echotexture distinct from that of bowel, so that they should also have sonographic features that differ from physiologically herniated bowel. ${ }^{3}$ Omphaloceles that contain only bowel are not uncommon, and although they are relatively small compared to those containing liver, generally they are large in comparison to physiologic herniation and will be easily differentiated from it unless they do not exceed the defined size criteria. ${ }^{12}$

Few reports of an omphalocele diagnosed in the first trimester have been published. ${ }^{2-4}$ In two instances the size criteria outlined herein for physiologic herniation were clearly exceeded, implying abnormality. Brown and colleagues ${ }^{2}$ diagnosed a case at 10.2 weeks with a $8 \times 9 \mathrm{~mm}$ homogeneous echogenic mass anterior to an abdomen of nearly the same dimension. Curtis and Watson ${ }^{3}$ reported an $18 \mathrm{~mm}$ omphalocele, which was larger than the abdominal circumference, at 12 weeks. Gray and coworkers ${ }^{4}$ described an omphalocele at 10.7 weeks that appeared similar to midgut herniation, but its size cannot be determined from the article, and whether or not it exceeds the size criteria for normal midgut herniation defined herein is not clear.

Some authors have suggested that after first trimester detection of any anterior wall mass, including probable physiologic midgut herniation, a repeat scan be obtained after either 12 or 14 weeks to confirm normalcy.1,4 The increasing number of early fetal sonograms in which midgut herniation will be detected makes this approach unduly cautious. It is suggested that, when an anterior wall mass fits the size and timing criteria defined by this study, no further evaluation is necessary.

Because variation exists in the assignment of gestational age on the basis of CRL, apparently due to the use of different data, we recommend that the CRL be substituted for gestational age when defining the timing for midgut herniation. This will remove a potential source of error in dating and allow stricter criteria for when midgut herniation should be expected.

In this study, no umbilical cord containing herniated midgut measured greater than $7 \mathrm{~mm}$ in any dimension. No fetus of CRL greater than $44 \mathrm{~mm}$ (or 
gestational age greater than 11.1 weeks) demonstrated herniated midgut. Confirmation that sonography can accurately detect the evolution of midgut cord herniation, and establishment of normal size criteria for the cord "mass," will allow differentiation from pathologic anterior wall masses except when their locus, size, and echo characteristics are identical to those described herein for midgut herniation.

Proposed guidelines for scanning the anterior abdominal wall are based on the timing and size findings of this study. For fetuses with a CRL measuring between 19 and $42 \mathrm{~mm}$, if the size criteria of the cord fit those described herein, it may safely be assumed that this represents normal physiologic herniation. A proximal cord mass exceeding $7 \mathrm{~mm}$ at any age is presumed abnormal and is deserving of follow-up scanning. Any cord mass present when CRL exceeds $44 \mathrm{~mm}$ (11.1 weeks) also is presumed abnormal and should be followed up.

\section{REFERENCES}

1. Cyr DR, Mack LA, Schoenecker SA, et al: Bowel migration in the normal fetus: US detection. Radiology 161: 119,1986

2. Brown DL, Emerson DS, Shulman LP, et al: Sonographic diagnosis of omphalocele during 10th week of gestation. AJR 153:825, 1989

3. Curtis JA, Watson L: Sonographic diagnosis of omphalocele in the first trimester of fetal gestation. J Ultrasound Med 7:97, 1988
4. Gray DL, Martin CM, Crane JP: Differential diagnosis of first trimester ventral wall defect. J Ultrasound Med $8: 255,1989$

5. Robinson HP, Fleming JEE: A critical evaluation of sonar "crown-rump length" measurements. Br J Obstet Gynaecol 82:702, 1975

6. Moore KL: The Developing Human: Clinically Oriented Embryology. Philadelphia, WB Saunders, 1988, p 228

7. Schmidt W, Yarkoni S, Crelin ES, et al: Sonographic visualization of physiologic anterior abdominal wall hernia in the first trimester. Obstet Gynecol 69:911, 1987

8. Green JJ, Hobbins JC: Abdominal ultrasound examination of the first-trimester fetus. Am J Obstet Gynecol 159:165, 1988

9. Timor-Tritsch IE, Warren WB, Peisner DB, et al: Firsttrimester midgut herniation: A high-frequency transvaginal sonographic study. Obstet Gynecol 161:831, 1989

10. Cullen MT, Green JJ, Reece EA, et al: A comparison of transvaginal and abdominal ultrasound in visualizing the first trimester conceptus. J Ultrasound Med 8:565, 1989

11. Guzman ER: Early prenatal diagnosis of gastroschisis with transvaginal ultrasonography. Am J Obstet Gynecol 162:1253, 1990

12. Nyberg DA, Fitzsimmons J, Mack LA, et al: Chromosomal abnormalities in fetuses with omphalocele. Significance of omphalocele contents. J Ultrasound Med 8+299, 1989 